\title{
Haemochromatosis presenting as congestive cardiac failure
}

\author{
Joanne Porter, Nathaniel Cary, Peter Schofield
}

\begin{abstract}
A 24 year old man with congestive cardiac failure was found to have grossly increased transferrin saturations, raised serum ferritin, and an iron-laden myocardium on biopsy. Initial treatment with the iron chelator desferrioxamine was replaced by weekly venesection. He was placed on the cardiac transplant list because of severe left ventricular dysfunction but was later removed because his symptoms and function improved. He remains well with few symptoms and is maintained on regular venesection and testosterone injections.
\end{abstract}

(Br Heart f 1995;73:73-75)

Keywords: haemochromatosis, venesection, heart failure

So-called genetic or hereditary haemochromatosis is an autosomal recessive disorder affecting iron metabolism. It occurs in $0 \cdot 1-0.5 \%$ of the population, with a possible gene frequency of up to $10 \%$, but the disorder often goes unrecognised. A significant association with the HLA-A3 antigen places the gene locus on the short arm of chromosome $6 .{ }^{1}$ The ten fold greater incidence in men is probably related to alcohol intake. Age is also an important factor with the disease becoming manifest mainly in middle age in men and after the menopause in women. Thus the disease is only seen in some homozygotes.

PATHOGENESIS

Inappropriately large quantities of iron are absorbed over many years and stores become saturated because excretory capacity is limited. Iron deposition in major organs causes hepatic cirrhosis, endocrine failure, skin pigmentation, diabetes mellitus, and cardiac impairment. The exact pathogenic mechanism remains to be determined but there is probably a direct toxic effect. Haemochromatosis can also be caused by a parenteral iron overload such as in regular transfusion for ineffective erythropoiesis in $\beta$ thalassaemia, by iron injections, and by an excessive oral intake. ${ }^{23}$

Early symptoms are non-specific, with lethargy, abdominal pain, and loss of libido being the most common presentations. Hepatic enlargement and skin pigmentation are common. Hepatic cirrhosis, diabetes mellitus, polyarthropathy, and hypogonadotrophic hypogonadism are seen. Cardiac involvement may take the form of arrhythmias, restrictive cardiomyopathy, or dilated cardiomyopathy. The most common cause of death is liver cancer, most frequently hepatocellular carcinoma associated with cirrhosis. If patients do present early, particularly men, then cardiac involvement is one of the major manifestations and without intensive treatment outcome is poor.

\section{INVESTIGATION}

Serum transferrin saturation, which is normally $16 \%-50 \%$, is the most accurate measure of iron overload. It can be estimated intermittently to follow progress. Serum ferritin is not accurate because concentrations may be normal despite organ iron overload. Serial computerised tomography scanning of the liver can be used to monitor hepatic iron content as can magnetic resonance imaging.

Specific cardiac investigation includes echocardiography or multigated acquisition (MUGA) scanning to document left ventricular function. Abnormalities can be seen echocardiographically before clinical cardiac involvement becomes apparent; with treatment there is an increase in ejection fraction and fractional shortening with reduced internal end systolic and end diastolic dimensions. ${ }^{4}$ Cardiac biopsy is a useful tool. Haemosiderin deposits show a bipolar distribution around the myocyte nucleus and can be confused with the age related pigment lipofuscin (figure). A Perls' stain is therefore mandatory if the diagnosis of haemochromatosis is not to be missed. Coronary angiography to exclude ischaemic cardiomyopathy may be appropriate.

\section{TREATMENT}

Supportive therapy for congestive cardiac failure is instituted in the immediate phase. Diuretics, angiotensin converting enzyme inhibitors, and anticoagulants form the basis of initial management. Once the exact diagnosis has been made other forms of treatment are introduced.

Venesection is the main form of treatment 


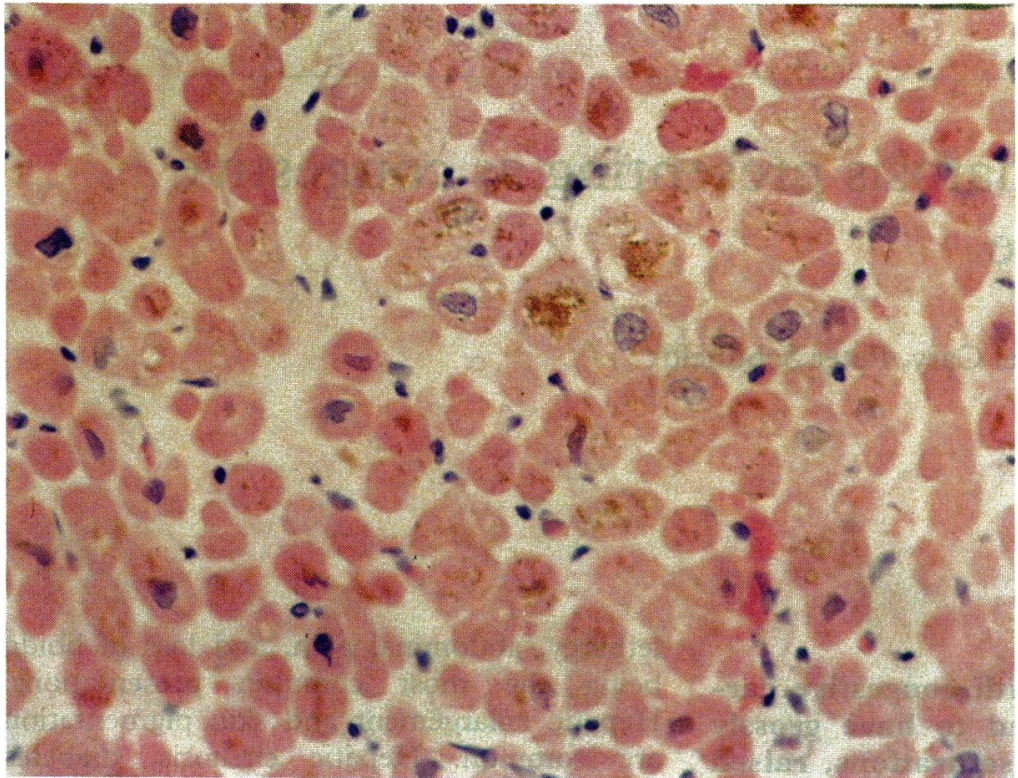

Endomyocardial biopsy specimen showing conspicuous deposits of haemosiderin brown stain within myocytes (Perls' Prussian blue reaction). (Medium power, haematoxylin and eosin.) desferrioxamine is poorly absorbed by mouth and therefore is given parenterally. Most commonly it is given overnight through the anterior abdominal wall by continuous subcutaneous infusion with a syringe driver pump.

Regular chelation therapy with desferrioxamine improved cardiac function in patients with secondary haemochromatosis as a result of transfusion-dependent thalassaemia. ${ }^{7}$ It protected such patients against the cardiac disease induced by iron overload. The coadministration of ascorbic acid increases the amount of chelatable iron and increases urinary excretion. Low doses of ascorbic acid do not cause the reported deterioration in cardiac function that is caused by higher doses.

In hereditary haemochromatosis, subcutaneous desferrioxamine is used in a daily dose of $25-60 \mathrm{mg} / \mathrm{kg}$ as a short term chelator when severe cardiac disease is life-threatening. Possible side effects are anaphylaxis, local erythema at the site of infusion, and ocular and auditory toxicity. Ocular complications include cataract formation and retinal photoreceptor alterations but these have mainly been seen at higher than recommended dosages. ${ }^{8}$ There is clearly a place for oral chelators given the difficulties of longterm administration of desferrioxamine. Clinical trials are underway with $\alpha$ ketohydroxypyridines, which because they are cheap to produce and orally effective are an attractive proposition in developing countries where thalassaemia is a significant problem.

Hormone replacement therapy for instance testosterone injections, is required for any endocrine deficiencies that are detected.

Screening of the affected family is vital to determine the mode of transmission and to identify by HLA studies, siblings or first degree relatives at risk. Physical examination and screening tests for disordered iron metabolism together with endocrine function and non-invasive imaging techniques are ideal. None the less, a liver biopsy is sometimes needed to establish the diagnosis of haemochromatosis beyond doubt. patients who had cardiomyopathy associate with haemochromatosis. Their condition improved or stabilised, whereas untreated patients had a worsening of their condition and a mean survival of one year after the appearance of cardiomyopathy.

It is important to realise that even when iron deficiency anaemia and hypoferritinaemia have appeared, myocardial iron stores are not depleted and treatment should be continued. Most patients will tolerate the removal of $500 \mathrm{ml}$ of blood weekly and some young individuals can have venesection twice a week in the initial phase. Intermittent courses of phlebotomy are required to prevent reaccumulation of iron. Five to eight units of blood should be removed annually, equivalent to $1-1.5 \mathrm{~g}$ of iron.

Chelation therapy is an additional tool that can be used in patients with haemochromatosis who require repeated transfusions and in whom venesection is not appropriate. Chelation therapy can also be used as an adjunct to venesection in acute cases of hereditary haemochromatosis. The chelator

\section{Case report}

A 24 year old man presented with a one week history of increasing shortness of breath, fatigue, and abdominal pain. He also had a cough, reduced appetite, and ankle swelling. On examination, he was unwell, pale, and mildly dyspnoeic at rest. His jugular venous pressure was raised to the angle of the jaw. His heart rate was 130 beats per minute in atrial fibrillation, blood pressure was $90 / 60$ $\mathrm{mm} \mathrm{Hg}$, and he had mitral regurgitation and a gallop rhythm. There were fine crackles throughout the chest, pitting oedema to the thigh, and hepatomegaly.

Initial blood. tests showed hepatic congestion. Chest $x$ ray showed cardiomegaly with pulmonary oedema and his electrocardiogram confirmed atrial fibrillation with non-specific $T$ wave changes laterally. An echocardiogram showed a dilated, poorly functioning left ventricle with a suggestion of apical thrombus 
and mitral regurgitation. Left ventricular ejection fraction measured by MUGA scan was $20 \%$.

Intravenous treatment with diuretics, inotropes, and heparin with oral digoxin improved his symptoms. Right heart catheterisation showed normal pressures with a mean pulmonary artery wedge pressure of $5 \mathrm{~mm}$ Hg. Right ventricular endomyocardial biopsy specimens showed conspicuous deposits of haemosiderin within cardiac myocytes with mild focal interstitial fibrosis but no evidence of myocarditis.

Further biochemical investigation showed

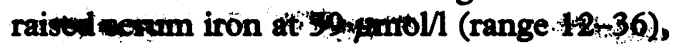
a grossly raised serum ferritin at over $3000 \mu \mathrm{g} /$ (range 15-235), and a transferrin saturation of $85 \%$ (range $16-50 \%$ ). This established the diagnosis of haemochromatosis, and treatment was started to reduce the iron load.

Desferrioxamine was given intravenously and then subcutaneously and regular venesection was introduced. Urinary iron was measured to ascertain that iron excretion was sufficient. Four months after an initial response he again deteriorated (ejection fraction $12 \%$ ) and he was assessed for a cardiac transplant. While he was on the transplant list, conservative treatment was continued and he improved to such an extent that his name was removed from the list. He is now almost symptom free and maintained on oral doses of frusemide, digoxin, lisinopril, warfarin, and folic acid with monthly testosterone injections. (Plasma gonadotrophins were much reduced because the anterior pituitary was involved). Regular venesection has improved ventricular function: the latest ejection fraction was $31 \%$.

1 Cox TM, Lord DK. Hereditary haemochromatosis. Eur 9 Haematol 1989;42:113-25.

2 Nichols GM, Bacon BR. Hereditary haemochromatosis: Pathogeneais and clinical features of a common disease. Am I Gastroenterol 1989;84:851-62.

3 Cox TM. Haemochromatosis. Blood Revieus 1990;4:75-87.

4 Candell-Riera J, Lu L, Seres I, Gonzalez JB, Batlle J, Permanyer-Miralda G, Garcia-del-Castillo $H$, SolerSoler J. Cardiac haemochromatosis: beneficial effects of iron removal thenopy. An echocardiographic study. $A m$ 9 Cardial 1983;52:824-9.

5 Bomford A, Williams $R$. Long term results of venesection therapy in idiopatbic haemochromatosis. Qf Med 1976; 45:611-23.

6 Finch SC, Finch CA. Idiopathic haemochromatosis and iron storage disease. Iron metabolism in haemochromatosis. Medicine 1955;34:381.

7 Davies SC, Hungerford IL, Arden GB, Marcus RE, Miller MH, Huehns BR. Ocular toxicity of high-dose intravenous desferrioxamine. Lancet 1983;ii:181-4.

8 Marcus RE, Davies SC, Bantock HM, Underwood SR, Walton S, Huehens BR. Desferrioxamine to improve cardiac function in iron-overloaded patients with thalassaemia major. Lancet 1984;i:392-3. 